\title{
Radiation effects on two-dimensional MHD Falkner-Skan wedge flow
}

\author{
M. Abdulhameed ${ }^{1, a}$, H. Saleh ${ }^{2, b}$, I. Hashim ${ }^{2, c}$ and R. Roslan ${ }^{1, d^{*}}$ \\ ${ }^{1}$ Centre for Research in Computational Mathematics, \\ Universiti Tun Hussein Onn Malaysia, \\ 86400 Parit Raja, Batu Pahat, Johor, Malaysia \\ ${ }^{2}$ School of Mathematical Sciences, Universiti Kebangsaan Malaysia, \\ 43600 Bangi Selangor, Malaysia \\ amoallahyidi@gmail.com, bdr.habibi@yahoo.com, cishak_h@ukm.my, rozaini@uthm.edu.my
}

Keywords: Falkner-Skan equation; Analytical solution; Boundary layer flow; MHD, Radiation

\begin{abstract}
Radiation effects on two-dimensional MHD Falkner-Skan boundary layer wedge have been studied. Analytical solution of nonlinear boundary-layer equations is obtained by modified homotopy perturbation method. It is observed that the magnetic field tends to decelerate fluid flow whereas radiations and thermal diffusion tend to increase fluid temperature.
\end{abstract}

\section{Introduction}

The problems arising from a thermal boundary-layer problem in the case of a two-dimensional incompressible flow passing over a wedge have been studied. These types of boundary-layer problems are expressed in the form of nonlinear third order partial differential equations, which cannot be solved directly in a closed form. These equations were first studied in [1]. The solutions for the case of the stream-wise pressure gradient parameter $\beta$ were first analyzed in [2]. The exact solutions of the Falkner-Skan boundary layer flow past a wedge placed symmetrically with respect to the flow direction was given in [3]. A finite-difference method for solving the Falkner-Skan equation was developed in [4]. Even though Falkner-Skan problem is a century old, it is still a topic of active current research. Recently, [5] solved the problem of the Falkner-Skan wedge flow by HPM-Pade' method. A new exact solutions ware developed in [6] for two-dimensional MHD Falkner-Skan boundary layer flow over a semi-infinite flat plate in the presence of a uniform magnetic field. Töpfer's algorithm and its extension was employed in [7] to solved Blasius and Falkner-Skan models. The main objective of the present investigation is to obtain an explicit analytic solution of the momentum and energy equations of MHD Falkner-Skan equation with thermal radiation using a new modified version of HPM. Here a new modified version of HPM is considered with a new set of auxiliary linear operator for both the velocity and temperature fields. In a recent article, [8] solved the non-Newtonian fluid flow problem for third-grade fluid .They used an approach similar to the one shown here; however, just the fully developed flow was analyzed.

\section{Formulation of the problem}

Consider the two-dimensional laminar boundary layer flow of a viscous incompressible electrically conducting fluid over a wedge. A constant magnetic field $B_{0}$ is applied in the $y$-direction and there is no external electric field. The induced magnetic field is neglected under the assumption of small magnetic Reynolds number. The temperature of the wall, $T_{w}$, is uniform and constant and is greater than the free stream temperature, $T_{\infty}$. Assumed that the free stream velocity $U_{\infty}$, is also uniform and constant. Further, assuming that the temperature changes resulting from viscous dissipation are small, the continuity equation and the boundary-layer equations may be expressed as:

$$
\begin{aligned}
& \frac{\partial u}{\partial x}+\frac{\partial v}{\partial y}=0 \\
& u \frac{\partial u}{\partial x}+v \frac{\partial v}{\partial y}=U(x) \frac{\partial U}{\partial X}+v \frac{\partial^{2} u}{\partial x^{2}}-\frac{\sigma B_{0}^{2}}{\rho}(u-U(x)),
\end{aligned}
$$




$$
\rho c_{p}\left(u \frac{\partial T}{\partial x}+v \frac{\partial T}{\partial y}\right)=k \frac{\partial^{2} T}{\partial y^{2}}-\frac{\partial q}{\partial y}+\mu\left(\frac{\partial u}{\partial y}\right)^{2},
$$

where $u$ and $v$ are the velocity components in the $x$ and $y$-directions respectively, $U(x)$ is the reference velocity at the edge of boundary layer and is a function of $x, \rho$ is the fluid density, $\mu$ is the kinematic viscosity, $\sigma$ is the electrical conductivity, $c_{p}$ is the specific heat at constant temperature, $k$ is the thermal conductivity of the fluid, $T$ is the temperature in the vicinity of the wedge and $q$ is the radiative heat flux. For an optically thick fluid [9], we can adopt Rosseland approximation, for radiative flux vector $q$. The radiative flux vector $q$ under Rosseland approximation becomes

$$
q=-\frac{4 \sigma^{*}}{3 k^{*}} \frac{\partial T^{4}}{\partial y}
$$

where $\sigma^{*}$ is the Stefan--Boltzmann constant and $k^{*}$ is the mean absorption coefficient. By Taylors' series about $T_{h}, T_{4}$ can be written as

$$
T^{4}=4 T_{h}^{3} T-3 T_{h}^{4} \text {. }
$$

Bu using Eqs. (4) and (5), Eq. (3) becomes

$$
\rho c_{p}\left(u \frac{\partial T}{\partial x}+v \frac{\partial T}{\partial y}\right)=\left(k+\frac{16 \sigma^{*} T_{h}^{3}}{3 k^{*}}\right) \frac{\partial^{2} T}{\partial y^{2}}+\mu\left(\frac{\partial u}{\partial y}\right)^{2} .
$$

The boundary conditions are as follows

$$
\begin{aligned}
& u=v=0 \text { and } T=T_{\infty} \quad \text { at } y=0, \\
& u=U(x)=U_{\infty}\left(\frac{x}{L}\right)^{m} \text { and } T=T_{\infty} \text { at } y \rightarrow \infty, \\
& u=U_{\infty} \text { and } T=T_{\infty}, \quad \text { at } x=0,
\end{aligned}
$$

where $U_{\infty}$ is the mean stream velocity, $L$ is the length of the wedge, $m$ is the Falkner-Skan powerlaw parameter, and $x$ is measured from the tip of the wedge. Introducing the stream function $\psi(x, y)$

$$
u=\frac{\partial \psi}{\partial y}, \quad v=-\frac{\partial \psi}{\partial x},
$$

and similarity variable:

$$
\begin{aligned}
\psi & =\sqrt{\frac{2 v x U(x)}{1+m}} f(\eta), \quad \eta=\sqrt{\frac{(1+m) U(x)}{2 v x}} y, \quad \beta=\frac{2 m}{m+1}, \\
\theta & =\frac{T-T_{w}}{T_{\infty}-T_{w}}, \quad M=\sqrt{\frac{2 \sigma}{\rho U_{\infty}(m+1)}} B_{0}, \quad \operatorname{Pr}=\frac{c_{p} \mu}{k}, \\
E c & =\frac{U x}{c_{p} T_{h}}, \quad R d=\frac{4 \sigma^{*} T_{h}^{3}}{k k^{*}}, \quad \operatorname{Re}=\frac{U}{v} .
\end{aligned}
$$

The resulting similarity equations from Eqs. (2) and (6) are

$$
\begin{aligned}
& \frac{d^{3} f}{d \eta^{3}}+f \frac{d^{2} f}{d \eta^{2}}+\beta\left[1-\left(\frac{d f}{d \eta}\right)^{2}\right]-M^{2}\left(\frac{d f}{d \eta}-1\right)=0, \\
& \left(1+\frac{4}{3} R d\right) \frac{d^{2} \theta}{d \eta^{2}}-\operatorname{PrRe} f \frac{d \theta}{d \eta}+\operatorname{Pr} E c\left(\frac{d^{2} f}{d \eta^{2}}\right)^{2}=0,
\end{aligned}
$$

The boundary condition (7) now give

$$
\begin{gathered}
f=\frac{d f}{d \eta}=\frac{d \theta}{d \eta}=0 \text { and } \theta=1 \text { at } \eta=0, \\
\frac{d f}{d \eta}=1 \text { and } \theta=0 \text { at } \eta \rightarrow \infty .
\end{gathered}
$$

where $M$ is the magnetic parameter, Re is the Reynolds number, Pr is the Prandtl number, $E c$ is the Eckert number and $R d$ is the radiation parameter. 


\section{New modified Homotopy perturbation solutions}

In this section, we will apply a new modified version of HPM to a nonlinear ordinary differential equation Eqs. (10) and (11). We introduce new set of auxiliary linear operator for a better accuracy We choose a new set of the auxiliary linear operators $L_{f}$ and $L_{\theta}$ in the form given below

$$
\begin{aligned}
& L_{f}(f)=\frac{d^{3} f}{d \eta^{3}}+\frac{d^{2} f}{d \eta^{2}}, \\
& L_{\theta}(\theta)=\frac{d^{2} \theta}{d \eta^{2}}+\frac{d \theta}{d \eta},
\end{aligned}
$$

We choose the auxiliary function $H(f, q)$ and $H(\theta, q)$ in the form:

$$
\begin{aligned}
& H(f, q)=(1-q)\left[\frac{d^{3} f}{d \eta^{3}}+\frac{d^{2} f}{d \eta^{2}}\right]+q\left\{\frac{d^{3} f}{d \eta^{3}}+f \frac{d^{2} f}{d \eta^{2}}+\beta\left[1-\left(\frac{d f}{d \eta}\right)^{2}\right]-M^{2}\left(\frac{d f}{d \eta}-1\right)\right\}, \\
& H(\theta, q)=(1-q)\left[\frac{d^{2} \theta}{d \eta^{2}}+\frac{d \theta}{d \eta}\right]+q\left\{\left(1+\frac{4}{3} R d\right) \frac{d^{2} \theta}{d \eta^{2}}-\operatorname{PrRe} f \frac{d \theta}{d \eta}+\operatorname{Pr} E c\left(\frac{d^{2} f}{d \eta^{2}}\right)^{2}\right\},
\end{aligned}
$$

Consider

$$
\begin{aligned}
& f(\eta)=f_{0}(\eta)+q f_{1}(\eta)+q^{2} f_{2}(\eta)+\ldots, \\
& \theta(\eta)=\theta_{0}(\eta)+q \theta_{1}(\eta)+q^{2} \theta_{2}(\eta)+\ldots,
\end{aligned}
$$

substituting $f$ and $\theta$ from Eqs. (17) and (18) into Eqs. (15) and (16) and some simplification and rearranging based on powers of $q$-terms, we have:

$$
\begin{aligned}
& q^{0}:\left\{\begin{array}{l}
\frac{d^{3} f_{0}(\eta)}{d \eta^{3}}+\frac{d^{2} f_{0}(\eta)}{d \eta^{2}}=0, \\
\frac{d^{2} \theta_{0}(\eta)}{d \eta^{2}}+\frac{d \theta_{0}(\eta)}{d \eta}=0,
\end{array}\right. \\
& f_{0}(0)=\frac{d f_{0}(0)}{d \eta}=\frac{d \theta_{0}(0)}{d \eta}=0, \quad \theta_{0}(0)=1, \quad \frac{d f_{0}(\infty)}{d \eta}=1, \quad \theta_{0}(\infty)=0, \\
& \int \frac{d^{3} f_{1}(\eta)}{d \eta^{3}}+\frac{d^{2} f_{1}(\eta)}{d \eta^{2}}=\frac{d^{3} f_{0}(\eta)}{d \eta^{3}}+\frac{d^{2} f_{0}(\eta)}{d \eta^{2}}+\frac{d^{3} f_{0}(\eta)}{d \eta^{3}}+f \frac{d^{2} f_{0}(\eta)}{d \eta^{2}}
\end{aligned}
$$

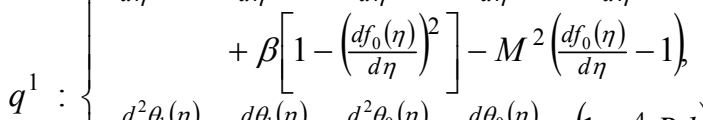

$$
\begin{aligned}
& \frac{d^{2} \theta_{1}(\eta)}{d \eta^{2}}+\frac{d \theta_{1}(\eta)}{d \eta}=\frac{d^{2} \theta_{0}(\eta)}{d \eta^{2}}+\frac{d \theta_{0}(\eta)}{d \eta}+\left(1+\frac{4}{3} R d\right) \frac{d^{2} \theta_{0}(\eta)}{d \eta^{2}} \\
& -\operatorname{Pr} \operatorname{Re} f \frac{d \theta_{0}(\eta)}{d \eta}+\operatorname{Pr} E c\left(\frac{d^{2} f_{0}(\eta)}{d \eta^{2}}\right)^{2}, \\
& f_{1}(0)=\frac{d f_{1}(0)}{d \eta}=\frac{d \theta_{1}(0)}{d \eta}=0, \quad \theta_{1}(0)=0, \quad \frac{d f_{1}(\infty)}{d \eta}=0, \quad \theta_{1}(\infty)=0, \\
& q^{2}:\left\{\begin{array}{c}
\frac{d^{3} f_{2}(\eta)}{d \eta^{3}}+\frac{d^{2} f_{2}(\eta)}{d \eta^{2}}=\frac{d^{3} f_{1}(\eta)}{d \eta^{3}}+\frac{d^{2} f_{1}(\eta)}{d \eta^{2}}+\frac{d^{3} f_{1}(\eta)}{d \eta^{3}} \\
+\left(f_{0} \frac{d^{2} f_{1}(\eta)}{d \eta^{2}}+f_{1} \frac{d^{2} f_{0}(\eta)}{d \eta^{2}}\right)-2 \beta \frac{d f_{0}(\eta)}{d \eta} \frac{d f_{1}(\eta)}{d \eta}-M^{2} \frac{d f_{1}(\eta)}{d \eta}, \\
\frac{d^{2} \theta_{2}(\eta)}{d \eta^{2}}+\frac{d \theta_{2}(\eta)}{d \eta}=\frac{d^{2} \theta_{1}(\eta)}{d \eta^{2}}+\frac{d \theta_{1}(\eta)}{d \eta}+\left(1+\frac{4}{3} R d\right) \frac{d^{2} \theta_{1}(\eta)}{d \eta^{2}} \\
-\operatorname{Pr} \operatorname{Re}\left(f_{0} \frac{d \theta_{1}(\eta)}{d \eta}+f_{1} \frac{d \theta_{0}(\eta)}{d \eta}\right)+2 \operatorname{Pr} E c \frac{d^{2} f_{0}(\eta)}{d \eta^{2}} \frac{d^{2} f_{1}(\eta)}{d \eta^{2}},
\end{array}\right. \\
& f_{2}(0)=\frac{d f_{2}(0)}{d \eta}=\frac{d \theta_{2}(0)}{d \eta}=0, \quad \theta_{2}(0)=0, \quad \frac{d f_{2}(\infty)}{d \eta}=0, \quad \theta_{2}(\infty)=0,
\end{aligned}
$$

Using Maple symbolic code, the solutions of Eqs. (19)-(24) is

$$
\begin{aligned}
f_{0}=e^{-\eta}+\eta-1, \\
\theta_{0}=1 \\
f_{1}=\frac{1}{4} e^{-2 \eta}\left\{-1+\beta-e^{2 \eta}\left(1+4 M^{2}+7 \beta\right)+2 e^{\eta}\left[1+3 \beta+4 \beta \eta+\eta^{2}+2 M^{2}(1+\eta)\right]\right\}
\end{aligned}
$$




$$
\begin{aligned}
& \theta_{1}=\frac{1}{2} e^{-2 \eta}\left(-1+e^{\eta}\right)^{2} \operatorname{Pr} E c, \\
f_{2}= & \frac{1}{72} e^{-3 \eta}\left\{5-9 \beta+4 \beta^{2}-e^{3 \eta}\left[197+144 M^{4}+657 \beta+460 \beta^{2}+27 M^{2}(13+19 \beta)\right]\right. \\
& +9 e^{\eta}\left[-11-2 \eta-2 \eta^{2}+4 \beta^{2}(3+2 \eta)+\beta\left(-3-6 \eta+2 \eta^{2}\right)+M^{2}(-1+\beta)(7+4 \eta)\right] \\
& +3 e^{2 \eta}\left[97+42 \eta+54 \eta^{2}+3 \eta^{4}+12 M^{2}(2+\eta)^{2}+4 \beta^{2}\left(29+42 \eta+12 \eta^{2}\right)\right. \\
& +3 \beta\left(77+74 \eta+14 \eta^{2}+8 \eta^{3}\right)+6 M^{2}\left[23+18 \eta+4 \eta^{2}+2 \eta^{3}\right. \\
& \left.\left.+\beta\left(25+30 \eta+8 \eta^{2}\right)\right]\right\}, \\
\theta_{2}= & \frac{1}{12} e^{-3 \eta} \operatorname{Pr} E c\left\{-4+2 \operatorname{Pr} \operatorname{Re}+4 \beta-e^{3 \eta}\left(-7+6 M^{2}+5 \operatorname{PrRe}+10 \beta\right)\right. \\
& +e^{\eta}\left[33+16 R d-3 \operatorname{PrRe}+6 \beta-6 \eta+6 \operatorname{PrRe} \eta+24 \beta \eta+6 \eta^{2}+6 M^{2}(1+2 \eta)\right] \\
& +2 e^{2 \eta}\left[8 R d(-1+\eta)+3\left[-6+4 \eta+\operatorname{Pr} \operatorname{Re}(1+\eta)^{2}\right]\right\},
\end{aligned}
$$

Substitution of Eqs. (25)-(30) into Eqs. (17) and (18) yields the second-order approximate solution for Eqs. (10) and (11):

\section{Analysis of results}

In order to see the physical insight of the considered problem, the influence of emerging parameters on the dimensionless velocity component and temperature are analyzed in this section. Figs. 1 and 2 demonstrate the influence of magnetic field on velocity component for the case of favourable pressure gradient (Fig. 1) and for the adverse pressure gradient (Fig 2). It is revealed from both Figs. 1 and 2 that velocity component decreases on increasing magnetic parameter $M$ in the boundary layer region. This implies that magnetic field decelerates fluid velocity. This is due to fact that the application of magnetic field to an electrically conducting fluid gives rise to resistive force which is known as Lorentz force. This force has tendency to decelerate fluid flow in the boundary layer region. It is noted that, the velocity component is more pronounce for the case of adverse pressure gradient. Fig. 3 demonstrates the effects of radiation on fluid temperature. It is observed from Fig. 3 that fluid temperature $\theta$ increases on increasing radiation parameter $R d$ in the boundary layer region which implies that radiation tends to enhance fluid temperature for infinite wedge flow. Fig. 4 illustrates the influence of Prandtl number Pr on fluid temperature. It is noticed from Fig. 4 that fluid temperature $\theta$ decreases on increasing $\operatorname{Pr}$ in the boundary layer region. Since Pr signifies the relative effects of viscosity to thermal conductivity. This implies that thermal diffusion tends to increase fluid temperature for infinite wedge flow.

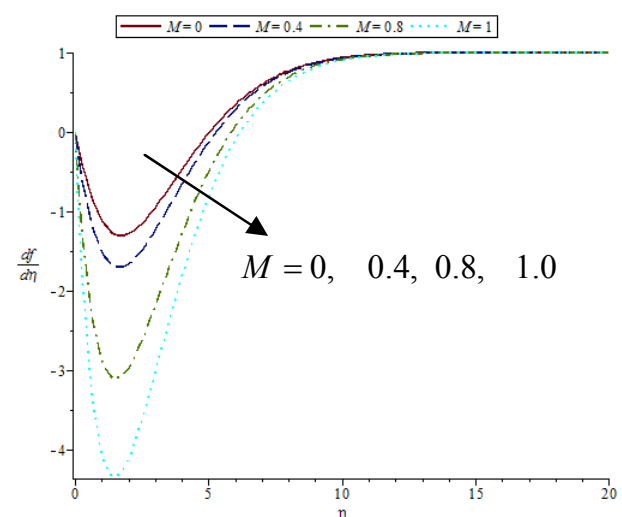

Fig. 1 Effects of $M$ on $\frac{d f}{d \eta}$ for the favourable pressure gradient $(\beta>0)$.

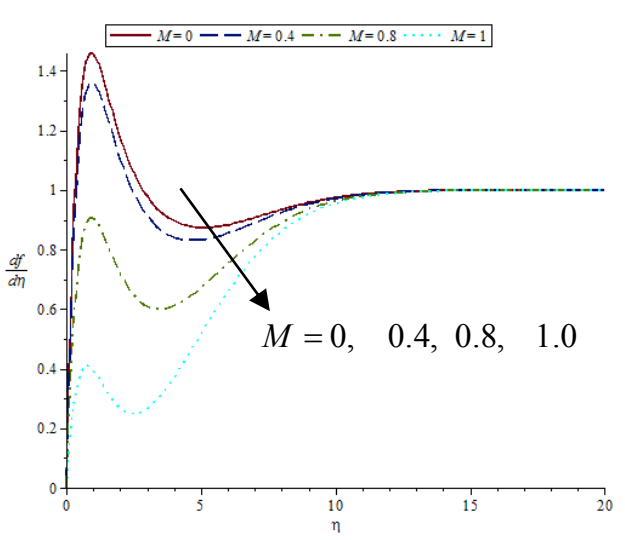

Fig. 2 Effects of $M$ on $\frac{d f}{d \eta}$ for the adverse pressure gradient $(\beta<0)$. 


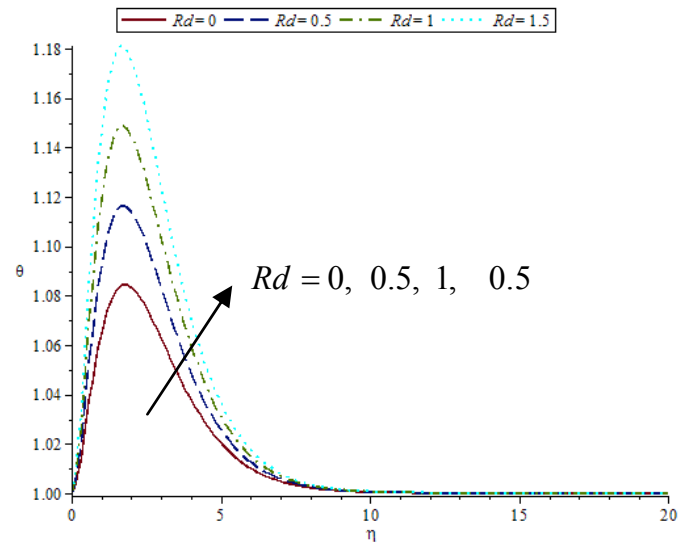

Fig. 3 Effects of $R d$ on $\theta$ for the favourable pressure gradient $(\beta>0)$ when $E c=\operatorname{Re}=0.3, \operatorname{Pr}=1, M=0.5$ and $\beta=1$ are fixed.

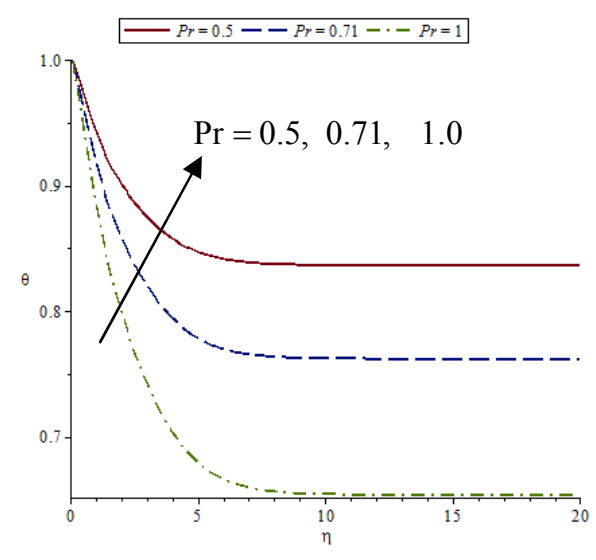

Fig. 4 Effects of $\operatorname{Pr}$ on $\theta$ for the favourable pressure gradient $(\beta>0)$ when $E c=\operatorname{Re}=0.3$, $R d=0.5, M=0.5$ and $\beta=1$ are fixed.

\section{Conclusions}

The flow and heat transfer arising from a thermal boundary-layer problem in the case of a twodimensional incompressible flow passing over a wedge have been studied. The nonlinear analysis is computed and results for velocity and temperature distributions are obtained using a new modified homotopy perturbation method. The results show that magnetic field tends to decelerate fluid flow while thermal diffusion and radiation tends to enhance fluid temperature

\section{References}

[1] V. M. Falkner, S.W. Skan, Some approximate solutions of the boundary layer equations, Philos. Mag. 12 (1931) 865-896.

[2] D.H. Hartree, On an equation occurring in Falkner and Skan's approximate treatment of the equations of the boundary layer. Proc. Cambr. Philos. Soc. 33 (1937) 223-239.

[3] K.R. Rajagopal, A. S. Gupta, T.Y. Na, A note on the Falkner-Skan flows of a non-

[4] Newtonian fluid. Int. J. Non-Linear Mech. 18 (1983) 313-320

[5] A. Asaithambi, A finite-difference method for the Falkner-Skan equation, Appl. Math. Comput. 92 (1998) 135-41.

[6] H. Bararnia, E. Ghasemi, S. Soleimani, A.R. Ghotbi, D.D. Ganji, Solution of the FalknerSkan wedge flow by HPM-Pade'method, Adv. Eng. Softw. 43(2012), 44-52.

[7] R.B. Kudenatti, S.R. Kirsur, L.N. Achala, N.M. Bujurke, Exact solution of two-dimensional MHD boundary layer flow over a semi-infinite flat plate, Commun. Nonlinear Sci. Numer. Simul. 18 (2013), 1151-1161.

[8] R. Fazio, Blasius problem and Falkner-Skan model: Töpfer's algorithm and its extension, Comput. Fluids, 73, (2013) 202-209.

[9] M. Abdulhameed, R. Roslan, M. Bin Mohamad, A modified homotopy perturbation transform method for transient flow of a third grade fluid in a channel with oscillating motion on the upper wall, J. Comput. Eng. 2014 (2014) 1-11.

[10] G. E. D. Azzam, Radiation effects on the MHD mixed free-forced convective flow past a semi-infinite moving vertical plate for high temperature differences, Phys. Scr. 66 (2002) 7176. 